\title{
Behavior-Rule Based Intrusion Detection Systems for Safety Critical Smart Grid Applications
}

\author{
Robert Mitchell and Ing-Ray Chen \\ Department of Computer Science \\ Virginia Tech \\ Email: \{rrmitche|irchen\}@vt.edu
}

\begin{abstract}
In this paper, a behavior-rule based intrusion detection system (BRIDS) is proposed for securing head-ends (HEs), distribution access points/data aggregation points (DAPs) and subscriber energy meters (SEMs) of a modern electrical grid in which continuity of operation is of the utmost importance. The impact of attacker behaviors on the effectiveness of a behavior-rule intrusion detection design is investigated. Using HEs, DAPs and SEMs as examples, it is demonstrated that a behavior-rule based intrusion detection technique can effectively trade false positives for a high detection probability to cope with sophisticated and hidden attackers to support ultra safe and secure applications. It is shown that BRIDS outperforms contemporary anomaly-based IDSs via comparative analysis.
\end{abstract}

keywords: Intrusion detection, cyber physical systems, security, safety, head-end, distribution access point, data aggregation point, subscriber energy meter.

\section{INTRODUCTION}

The most prominent characteristic of a smart grid such as a modern electrical grid or electricity infrastructure is the feedback loop that acts on the physical environment. In other words, the physical environment provides data to the sensors attached to the Wide Area Networks (WANs), Neighborhood Area Networks (NANs) and Home Area Networks (HANs) whose data feed the control units in the production, transmission, distribution and consumption segments that drive the actuators which change the physical environment. Modern electricity infrastructure is often characterized by sophisticated reliability, efficiency, sustainability and utility control units interacting with the physical environment including subscriber appliances. This paper concerns intrusion detection mechanisms for detecting compromised devices embedded in WANs, NANs and HANs for supporting safe and secure applications that subscribers can depend on with confidence.

Intrusion detection system (IDS) techniques for this domain are still in their infancy with very little work reported in the literature. Only [2], [3], [6], [10], [13], [14], [16], [19], [20], [21], [22], [23] reported related intrusion detection. However, nine of these had no numerical data regarding the false negative probability $p_{\text {fn }}$ (i.e., missing a bad node) and the false positive probability $p_{\mathrm{fp}}$ (i.e., misidentifying a good node as a bad node). The other three had minimal numerical data: one or two data points characterizing $p_{\mathrm{fn}} / p_{\mathrm{fp}}$ instead of a dataset that could be transformed into a Receiver Operating Characteristic (ROC) plot, i.e., a $p_{\text {fn }}$ vs. $p_{\text {fp }}$ curve that describes the relationship between $p_{\mathrm{fn}}$ and $p_{\mathrm{fp}}$ obtained as a result of applying IDS techniques.

Specifically, Zhang et al. [22], [23] studied two detection algorithms called CLONALG and AIRS2Parallel. CLONALG is unsupervised. AIRS2Parallel is semi-supervised. They reported that CLONALG had a detection accuracy between $80.1 \%$ and $99.7 \%$ and AIRS2Parallel had an accuracy between $82.1 \%$ and $98.7 \%$, where the detection accuracy is the likelihood that a node is classified correctly, calculated by $1-p_{\mathrm{fp}}-p_{\mathrm{fn}}$. He and Blum [10] investigated a series of anomaly-based IDSs including Locally Optimum Unknown Direction (LOUD), Locally Optimum Estimated Direction (LOED), LOUD-Generalized Likelihood Ratio (LOUD-GLR) and LOED-Generalized Likelihood Ratio (LOED-GLR). He and Blum's LOUD-GLR approach performed the best: The maximum detection rate (i.e., $1-p_{\text {fn }}$ ) is reportedly $95 \%$. However, no ROC data were given in [10], [22], [23].

Intrusion detection techniques in general can be classified into three types: signature-based, anomaly-based and specification-based techniques. In this paper, specificationbased detection is considered rather than signature-based detection to deal with unknown attacker patterns. Specificationbased techniques are considered rather than anomaly-based ones (such as those by Zhang et al. [22], [23] and He and Blum [10]) to avoid using resource constrained sensors or actuators in a WAN for profiling anomaly patterns (for example, through learning) and to avoid high false positives (treating good nodes as bad nodes).

To accommodate resource constrained devices, this paper develops the design notion of behavior rules for specifying acceptable behaviors of physical devices in a WAN, NAN or HAN. Rule-based intrusion detection thus far has been applied only in the context of communication networks which have no concern of physical environments and the closed-loop control structure as in a head-end (HE), distribution access point/data aggregation point (DAP) or subscriber energy meter (SEM).

In the literature, specification-based IDS techniques have been proposed for intrusion detection of communication protocol misbehaving patterns [7], [8], [9], [12]. Da Silva et al. [8] propose an IDS that applies seven types of traffic-based rules to detect intruders: interval, retransmission, integrity, delay, repetition, radio transmission range and jamming. Ioannis et al. [12] propose a multitrust IDS with traffic-based collection that audits the forwarding behavior of suspects to detect blackhole and greyhole attacks launched by captured devices 
based on the the rate (versus the count) of specification violations. [7], [9] also only considered specification-based state machines for intrusion detection of misbehaving patterns in communication networks. The specification-based technique in this paper distinguishes itself from [7], [8], [9], [12] cited above by addressing the unique requirements of the domain. First, modern electricity infrastructure has control loops that tie the physical environment to the CPS. Second, components are stationary which eliminates IDSs based on instantaneous motion or movement profiles. Third, they are federated systems; bulk power generators, energy markets, transmission providers, distribution providers and subscribers own, host and operate different segments of the CPS. Fourth, their scale is substantial; for example, the count of SEMs could be in the millions. Fifth, these CPSs are heterogeneous. In this work, specification-based behavior rules are derived from control loops which tie the intrusion detection to the critical business rules of the CPS while not relying on motion or track data used in other approaches. Also, the goals of each interest in the CPS are considered in forming behavior rules: bulk power generators want full utilization, energy markets want to match supply and demand, microgrids want to optimize sustainability or reliability and customers want to minimize cost. To address scalability, the state machines are pruned and tunable audit frequencies are provided. Three node types are considered to account for heterogeneity in the CPS.

The contribution of our work relative to prior work cited is that behavior rules for WAN, NAN and HAN devices controlling actuators and sensors embedded in the physical environment are specifically considered. Further, a method to transform behavior rules to a state machine is proposed, so that a device that is being monitored for its behavior can be checked against the transformed state machine for deviation from its behavior specification. Untreated in the literature [17], in this paper the impact of attacker behaviors on the effectiveness of intrusion detection in the production, transmission, distribution and consumption segments is also investigated. Using HEs, DAPs and SEMs as examples, it is demonstrated that an intrusion detection technique can effectively trade false positives for a high detection probability to cope with more sophisticated and hidden attackers to support ultra safe and secure applications. Moreover, it is shown that a behavior-rule based intrusion detection system (BRIDS) design outperforms contemporary anomaly-based IDSs [10], [22], [23] via comparative analysis.

\section{Model AND Design}

\section{A. System Model}

a) Reference System: A modern electrical grid cyber physical system (CPS) embedding physical components is considered as the reference model as illustrated in Figure 1. For ease of disposition, this paper is particularly concerned with three types of physical devices: HEs, DAPs and SEMs. Many examples exist with these three devices. Figure 1 shows their hierarchical relationship: The scope of an HE, which is operated by a bulk power generator or energy market, encompasses many DAPs, which are operated by the

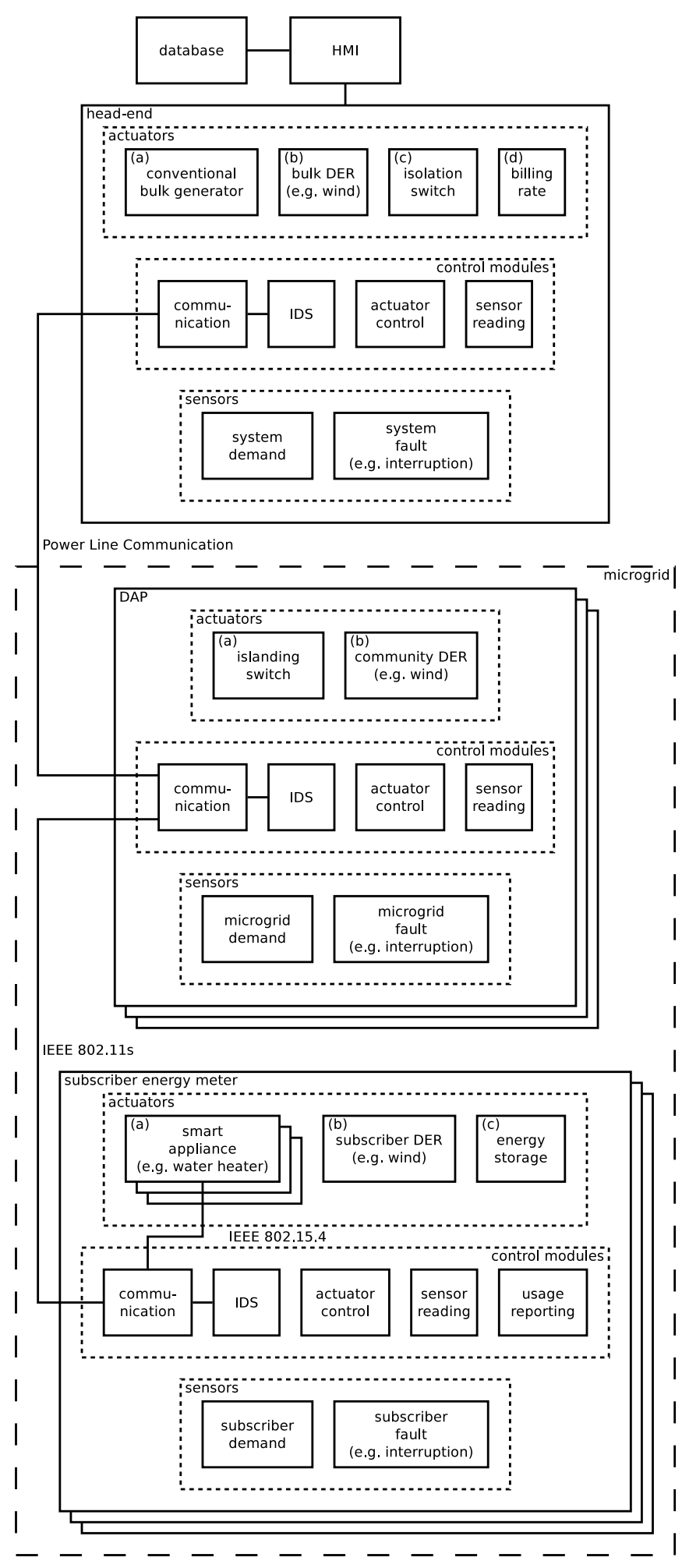

Fig. 1. Modern Electrical Grid CPS. 
transmission or distribution providers. The scope of a DAP encompasses many SEMs, which are hosted by subscribers (residential, commercial or industrial). This figure also shows the control modules running at each node: Host and system IDS modules run on every HE, DAP and SEM. Host IDS modules are loaded with the state machines pertaining to the relevant trustees. For example, HE host IDSs include HE and DAP state machines while SEM host IDSs include SEM and DAP state machines. Figure 1 illustrates the control modules, actuators and sensors that relate to the IDS design and how it integrates with the existing communications infrastructure. Power Line Communication links HEs to DAPs, IEEE 802.11s Wireless Mesh Networking links DAPs to SEMs and IEEE 802.15.4 Wireless Personal Area Networking links SEMs to smart appliances and customer distributed energy resources (DERs). DERs are alternatives to the bulk power generators. Including capital investment, fuel and consumables for both, the cost per Watt for DERs is typically higher. However, DERs (e.g., wind generators, geothermal units or solar cells) surpass bulk power generators (e.g., coal or nuclear-powered) in sustainability. While they are not advantageous in terms of sustainability, hydrocarbon-based (in addition to the renewable) DERs provide redundancy in case of breakage in the transmission network. Members of a microgrid can pool resources to buy and operate a community DER or individual subscribers can go it alone. A database collects, stores and distributes data from sensors. A human machine interface (HMI) allows an operator to control the system and view its status using sensor data in the colocated database. Figure 1 shows two actuators for the HE: a bulk generator and isolation switches. The bulk generator may come in the form of a largecapacity power station consuming hydrocarbon or fissile fuel. Isolation switches open and close circuits in the transmission network due to faults or maintenance. Figure 1 shows two actuators for the DAP: an islanding switch and a community DER. The islanding switch separates and joins a microgrid with the transmission network due to faults or maintenance. A community DER may come in the form of a medium-scale wind generator, geothermal unit or solar cell array. Figure 1 shows two actuators for the SEM: a smart appliance and a subscriber DER. A smart appliance tailors its duty cycle (e.g., compressor active/idle ratio for an HVAC unit) or scheduling (e.g., start time for a dishwasher) based on microgrid demand and billing rate. A community DER may come in the form of a small-scale wind generator, geothermal unit or solar cell array. Figure 1 shows two sensors for each of the HE, DAP and SEM which detect demand (Watts) and faults (derived from phase of the $\mathrm{AC}$ waveform) at the system, microgrid and subscriber levels, respectively.

b) Behavior Monitoring: Our IDS approach is based on behavior monitoring. A neighbor HE, DAP or SEM is used to monitor (specifically, measure the compliance degree of) one or more trustees of different types. DAPs are less resource rich than an HE due to high volume and tight size, weight and power constraints. However, they are plentiful which results in significant aggregate time and space that can be accumulated to monitor other DAPs or SEMs. An SEM monitors other neighboring SEMs only due to a high degree of resource constraints.

c) Threat Model: The threat model explains possible attacks performed by a compromised device (HE, DAP or SEM), which will cause its behavior to deviate from good behaviors specified by a set of behavior rules used by the IDS. Two attacker archetypes are differentiated: reckless and random. A reckless attacker performs attacks whenever it has a chance. The main objective is to impair the functionality at the earliest possible time. A random attacker, on the other hand, performs attacks only randomly to avoid detection. It is thus insidious and deceptive with the objective to cripple the functionality. The attacker behavior is modeled by a random attack probability $p_{a}$. When $p_{a}=1$ the attacker is a reckless adversary. Imperfect monitoring is modeled by an error parameter, $p_{\text {err }}$, representing the probability of a monitor node misidentifying the status of the trustee node due to ambient noise, temporary system faults, and/or wireless communication faults in the environment. In general a node may deduce $p_{\text {err }}$ at runtime by sensing the amount of ambient noise, system errors, and/or wireless communication errors around it.

\section{B. Problem Definition}

We define the problem to be solved in the context of Figure 1. Broadly, the problem we are trying to solve is the vulnerability to infrastructure damage, service interruption and revenue loss caused by malicious actors. We aim to provide a solution to this problem by detecting malicious devices that exploit the vulnerability through known or unknown attacks. The solution we are offering is a behavior-rule based design with which misbehavior of a device manifested as a result of attacks exploiting the vulnerability exposed may be detected, regardless of whether the attack is known or unknown. In the context of the electrical power grid in Figure 1, we aim to solve this problem by detecting malicious devices, including HE, DAP and SEM devices. For example, an opportunistic vandal could completely unfurl the blades of a wind DER during high wind conditions to damage the apparatus. A state sponsored attacker could open the isolation switches at a bulk energy provider to disrupt the service to the utilitys customers. A disgruntled insider at a bulk energy provider could lower the billing rate to cause the enterprise to lose money on all power it sold at the artificially depressed rate. A frugal subscriber could modify the usage reporting module of their subscriber energy meter to reduce their financial obligation to the energy provider. Regardless of the form of attacks, we aim to provide a solution for malicious device detection that is accurate in detection rate (close to 100\%) while limiting the false positive probability to a minimum (e.g., less than $10 \%$ ).

\section{Behavior Rules}

Our IDS design for the reference model relies on the use of lightweight specification-based behavior rules for each device. They are oriented toward detecting an inside attacker attached to a specific physical device, providing a continuous output between 0 and 1 (while accounting for transient faults and human errors) and allowing a monitor to perform intrusion 
TABLE I

HE BEHAVIOR RULES

\begin{tabular}{|l|l|l|}
\hline Description & Trustee & Monitor \\
\hline \hline $\begin{array}{l}\text { Turn off appliance block (for example, all } \\
\text { water heaters in a microgrid) if system } \\
\text { demand is above threshold }\end{array}$ & $\mathrm{HE}$ & $\mathrm{HE}$ \\
\hline $\begin{array}{l}\text { Decrease duty cycle (t/T, where } \\
\mathrm{t}=\text { pulse width and T } \mathrm{T} \text { period) for appliance } \\
\text { block if system demand is above threshold }\end{array}$ & $\mathrm{HE}$ & $\mathrm{HE}$ \\
\hline $\begin{array}{l}\text { Turn on appliance block if system demand is } \\
\text { below threshold }\end{array}$ & $\mathrm{HE}$ & $\mathrm{HE}$ \\
\hline $\begin{array}{l}\text { Increase duty cycle }(\mathrm{t} / \mathrm{T}, \text { where } \\
\mathrm{t}=\text { pulse width and } T=\text { period) for appliance } \\
\text { block if system demand is below threshold }\end{array}$ & $\mathrm{HE}$ & $\mathrm{HE}$ \\
\hline $\begin{array}{l}\text { Increase billing rate if system demand is above } \\
\text { threshold }\end{array}$ & $\mathrm{HE}$ & $\mathrm{HE}$ \\
\hline $\begin{array}{l}\text { Decrease billing rate if system demand is } \\
\text { below } \mu_{d}-\epsilon_{d}\end{array}$ & $\mathrm{HE}$ & $\mathrm{HE}$ \\
\hline Close isolation switch if no fault or maintenance & $\mathrm{HE}$ & $\mathrm{HE}$ \\
\hline $\begin{array}{l}\text { Connect DER to distribution segment if system } \\
\text { demand is above } \mu_{d}+\epsilon_{d}\end{array}$ & $\mathrm{HE}$ & $\mathrm{HE}$ \\
\hline $\begin{array}{l}\text { If fault sensors indicate an interruption, notify } \\
\text { affected nodes }\end{array}$ & $\mathrm{HE}$ & $\mathrm{HE}$ \\
\hline
\end{tabular}

TABLE II

DAP BEHAVIOR RULES

\begin{tabular}{|l|l|l|}
\hline Description & Trustee & Monitor \\
\hline \hline $\begin{array}{l}\text { Request subscriber load decrease if subscriber } \\
\text { demand is above } \mu_{d}+\epsilon_{d}\end{array}$ & DAP & DAP, HE \\
\hline $\begin{array}{l}\text { Request subscriber load increase if subscriber } \\
\text { demand is below } \mu_{d}-\epsilon_{d}\end{array}$ & DAP & DAP, HE \\
\hline $\begin{array}{l}\text { Open island switch if bulk generation is } \\
\text { interrupted }\end{array}$ & DAP & DAP, HE \\
\hline Relay packets & DAP & DAP, HE \\
\hline Don't source (replay or inject) packets & DAP & DAP, HE \\
\hline Use community DER generators if available & DAP & DAP, HE \\
\hline $\begin{array}{l}\text { If fault sensors indicate an interruption, } \\
\text { notify affected nodes }\end{array}$ & DAP & DAP, HE \\
\hline $\begin{array}{l}\text { If demand above threshold, increase pitch of } \\
\text { (unfurl) wind DER generator to maximize } \\
\text { power }\end{array}$ & DAP & DAP, HE \\
\hline $\begin{array}{l}\text { If demand below threshold, decrease pitch of } \\
\text { (furl) wind DER generator to maximize lifetime }\end{array}$ & DAP & DAP, HE \\
\hline
\end{tabular}

detection on a neighbor trustee through monitoring. Here a monitor is itself a physical device with capability to do intrusion detection on trustee nodes assigned to it. For example, an SEM may monitor another SEM within radio range. An HE may monitor other HE or DAP trustee devices within radio range. Therefore, an HE might have several sets of behavior rules (and thus several state machines), one for each trustee.

Tables I, II and III list the behavior rules for the HE, DAPs and SEMs. These tables specify the trustee and monitor devices for applying the IDS technique.

The networking concepts used in the behavior rules include: Packets received are the inbound protocol data units handled by the communications subsystem or application on a node; they are measured with frequency $(\mathrm{Hz})$ with a domain of 0 to 10 packets per second. A node receives packets for which it is not the intended receiver, but possibly is a waypoint on the path to the destination. The communications subsystem drops these packets or relays them. Packets forwarded counts these packets the communications subsystem passes along using frequency $(\mathrm{Hz})$ over the same domain as packets received. Packet sourcing is when an application generates a protocol
TABLE III

SEM BEHAVIOR RULES

\begin{tabular}{|l|l|l|}
\hline Description & Trustee & Monitor \\
\hline \hline Generate usage data periodically & SEM & SEM, DAP \\
\hline $\begin{array}{l}\text { Deactivate time independent appliances if } \\
\text { billing rate is above } \mu_{r}+\epsilon_{r}\end{array}$ & SEM & SEM, DAP \\
\hline $\begin{array}{l}\text { Activate time independent appliances or store } \\
\text { energy if billing rate is below } \mu_{r}-\epsilon_{r}\end{array}$ & SEM & SEM, DAP \\
\hline $\begin{array}{l}\text { Deactivate time independent appliances if } \\
\text { microgrid demand is above } \mu_{d}+\epsilon_{d}\end{array}$ & SEM & SEM, DAP \\
\hline $\begin{array}{l}\text { Activate time independent appliances or store } \\
\text { energy if microgrid demand is below } \mu_{d}-\epsilon_{d}\end{array}$ & SEM & SEM, DAP \\
\hline Use subscriber DERs if available & SEM & SEM, DAP \\
\hline $\begin{array}{l}\text { If fault sensors indicate an interruption, } \\
\text { notify affected nodes }\end{array}$ & SEM & SEM, DAP \\
\hline $\begin{array}{l}\text { If subscriber demand above threshold, } \\
\text { increase pitch of (unfurl) wind DER } \\
\text { generator to maximize power }\end{array}$ & SEM & SEM, DAP \\
\hline $\begin{array}{l}\text { If subscriber demand below threshold, } \\
\text { decrease pitch of (furl) wind DER generator } \\
\text { to maximize lifetime }\end{array}$ & SEM & SEM, DAP \\
\hline
\end{tabular}

data unit and passes it down to the communications subsystem for transmission. A good node populates these packets with legitimate sensor or status data, but a bad node populates these packets with corrupt sensor or status data or replays of previously received packets. $\epsilon_{f}$ is a threshold for the difference between packets received and packets forwarded. The networking condition is an abbreviation of packets received and forwarded used to manage the size of the behavior rule state machine. $\mu_{d}$ is the nominal power demand. $\epsilon_{d}$ is a distance from $\mu_{d}$ beyond which a control algorithm should take action to match power supply with demand. $\mu_{r}$ is the nominal billing rate. $\epsilon_{r}$ is a distance from $\mu_{r}$ beyond which a control algorithm should take action to capitalize on a low billing rate or avoid consuming at a high one.

Our behavior-rule specification-based technique approaches the intrusion detection problem from the behavior/evidence domain compared with signature-based techniques that approach the problem from the attacker domain. Hence, the patterns by which an attacker performs attacks and "how" an attacker performs attacks do not need to be known. Rather, a monitor device simply checks the behavior of a trustee device manifested from evidence of compliance/deviation against "good" and "bad" behaviors specified by a set of behavior rules for that device. Our approach thus can address all potential attacks, known or unknown. We claim behavior rule-based detection is able to cope with unknown attacks because all attacks lead to behavior anomaly. This capability is similar to anomaly detection which, unlike signature-based detection, can cope with zero-day attacks. Nevertheless, if the rule set is incomplete, that is, if the specification of anticipated behavior is incomplete, it is possible misbehavior manifested as a result of known or unknown attacks will be missed, and, consequently, the attacker will be undetected.

\section{Transforming Rules to State Machines}

Each behavior rule does not specify just one attack state, but a number of states, some of which are good states in which good behavior (obedience of this behavior rule) is observed, while others are bad states in which bad behavior (violation 
of this behavior rule) is observed. A behavior rule thus has a number of state variables, each with a range of values, together indicating whether the node is in good or bad behavior status (with respect to this rule). A device (HE, DAP or SEM), on the other hand, has a number of behavior rules; thus, it is possible that the state variables for one rule have intersections with those in another rule if they have the same logical clause. For example, the "system demand" state variable appears in HE rules 1-5 and 7. In this case, only one state variable will be used in these six rules to represent the "system demand" status. At the end, the underlying state machine for the behavior rule set of a device (e.g., Table I for HE) will consist of a set of unique state variables common to all behavior rules (e.g., system demand in HE rules 1-5 and 7) together indicating whether a device is in a good or bad behavior state (reflecting all behavior rules).

The following procedure transforms a behavior specification into a state machine: First, the "bad behavior indicator" as a result of a behavior rule being violated is identified. Then, this bad behavior indicator is transformed into a conjunctive normal form predicate and the involved state components in the underlying state machine are identified. Next, for each device (that is, an HE, DAP or SEM), the bad behavior indicators are combined into a Boolean expression in disjunctive normal form. Then, the union of all predicate variables is transformed into the state components of a state machine and their corresponding ranges are established. Finally, the number of states is managed by state collapsing and identifying combinations of values that are not legitimate. How a state machine is derived from the behavior specification in terms of behavior rules for the reference model is exemplified below.

a) Identify Bad Behavior Indicators: Attacks performed by a compromised sensor/actuator will drive the HE, DAP or SEM into certain bad behavior indicators identifiable through analyzing the specification-based behavior rules.

For the HE device, there are nine bad behavior indicators as a result of violating the nine behavior rules for HEs listed in Table I. The first HE bad behavior indicator is that the HE activates a block of appliances but the system demand is above some threshold. The second HE bad behavior indicator is that the HE increases the duty cycle for a block of appliances but the system demand is above some threshold. The third HE bad behavior indicator is that the HE deactivates a block of appliances but the system demand is below some threshold. The fourth HE bad behavior indicator is that the HE decreases the duty cycle for a block of appliances but the system demand is below some threshold. The fifth HE bad behavior indicator is that the HE decreases the billing rate but the system demand is above some threshold. The sixth HE bad behavior indicator is that the HE increases the billing rate but the system demand is below some threshold. The seventh HE bad behavior indicator is that the HE opens the switch for a microgrid but there is no associated fault or maintenance. The eighth HE bad behavior indicator is that DERs are disconnected but the system demand is above some threshold. The ninth HE bad behavior indicator is that an interruption is present but the HE has not generated an alert. For all of these HE bad behavior indicators, the HE is the trustee and all DAPs are monitors.
For the DAP device, there are eight bad behavior indicators as a result of violating the nine behavior rules for DAPs listed in Table II. The first DAP bad behavior indicator is that the $\mathrm{HE}$ requests a load increase, but microgrid demand is above some threshold. The second DAP bad behavior indicator is that the HE requests a load decrease, but microgrid demand is below some threshold. For these first two DAP bad behavior indicators, the HE is the trustee, and a DAP is the monitor. The third DAP bad behavior indicator is that the microgrid is islanded, but there is no interruption. For the third DAP bad behavior indicator, a DAP is the trustee, and the HE is the monitor. The fourth DAP bad behavior indicator is that the number of packets forwarded by the DAP does not equal the number of packets received by the DAP. This rule corresponds with the two behavior rules concerning packet handling. For the fourth DAP bad behavior indicator, a DAP is the trustee, and the HE and SEMs are monitors. The fifth DAP bad behavior indicator is that the community DER is not connected, but it is available. The sixth DAP bad behavior indicator is that an interruption is present, but the DAP has not generated an alert. The seventh DAP bad behavior indicator is that the DAP decreases the pitch of wind DER generator blades, but the microgrid demand is above some threshold. The eighth DAP bad behavior indicator is that the DAP increases the pitch of wind DER generator blades, but the microgrid demand is below some threshold. For the fifth through eighth DAP bad behavior indicators, a DAP is the trustee, and the $\mathrm{HE}$ is the monitor.

For the SEM device, there are nine bad behavior indicators as a result of violating the nine behavior rules for SEMs listed in Table III. The first SEM bad behavior indicator is that the SEM is not generating usage data. The second SEM bad behavior indicator is that time-independent smart appliances are active, but the billing rate is above some threshold. The third SEM bad behavior indicator is that the subscriber is not banking electricity, but the billing rate is below some threshold. The fourth SEM bad behavior indicator is that timeindependent smart appliances are active, but the demand is above some threshold. The fifth SEM bad behavior indicator is that the subscriber is not banking electricity, but the demand rate is below some threshold. The sixth SEM bad behavior indicator is that the subscriber DER is not connected, but it is available. The seventh SEM bad behavior indicator is that an interruption is present, but the SEM has not generated an alert. The eighth SEM bad behavior indicator is that the SEM decreases the pitch of wind DER generator blades, but the subscriber demand is above some threshold. The ninth SEM bad behavior indicator is that the SEM increases the pitch of wind DER generator blades, but the subscriber demand is below some threshold. For all of these SEM bad behavior indicators, an SEM is the trustee, and the DAP is the monitor.

b) Express Bad Behavior Indicators in Conjunctive Normal Form: Tables IV, V and VI list the bad behavior indicators in Conjunctive Normal Form for HE, DAP and SEM nodes, respectively.

c) Consolidate Predicates in Disjunctive Normal Form: Each type of device (HE, DAP or SEM) has a distinct behavior rule set based on its specific control modules, actuators and 
TABLE IV

HE BAD BEHAVIOR INDICATORS IN CONJUNCTIVE NORMAL FORM

$($ Appliance Block $=$ ACTIVE $) \wedge\left(\right.$ System Demand $\left.>\mu_{d}+\epsilon_{d}\right)$

(New Appliance Duty Cycle $>$ Old Appliance Duty Cycle)

$\wedge$ (System Demand $\left.>\mu_{d}+\epsilon_{d}\right)$

$\left(\right.$ Appliance Block $=$ INACTIVE) $\wedge\left(\right.$ System Demand $\left.<\mu_{d}-\epsilon_{d}\right)$

(New Appliance Duty Cycle $<$ Old Appliance Duty Cycle)

$\wedge$ (System Demand $<\mu_{d}-\epsilon_{d}$ )

(New Billing Rate $<$ Old Billing Rate) $\wedge$ (System Demand $\left.>\mu_{d}+\epsilon_{d}\right)$

(New Billing Rate $>$ Old Billing Rate) $\wedge$ (System Demand $\left.<\mu_{d}-\epsilon_{d}\right)$

$($ Isolation Switch Position $=$ OPEN $) \wedge($ Fault $=$ FALSE $)$

$\wedge$ (Maintenance $=$ FALSE)

$($ DER $=$ DISCONNECTED $) \wedge\left(\right.$ System Demand $\left.>\mu_{d}+\epsilon_{d}\right)$

$($ Interruption $=\mathrm{TRUE}) \wedge($ Alert $=\mathrm{NULL})$

TABLE V

DAP BAD BEHAVIOR INDICATORS IN CONJUNCTIVE NORMAL FORM

\begin{tabular}{|l|}
\hline (New Load Request $>$ Old Load Request $)$ \\
$\wedge$ (Microgrid Demand $\left.>\mu_{d}+\epsilon_{d}\right)$ \\
\hline (New Load Request $<$ Old Load Request $)$ \\
$\wedge$ (Microgrid Demand $\left.<\mu_{d}-\epsilon_{d}\right)$ \\
\hline (Island Switch Position $=$ OPEN) $\wedge$ (Interruption $=$ TRUE) \\
\hline $\mid$ Forwarded Packets - Received Packets $>\epsilon_{f}$ \\
\hline (DER Connection $=$ FALSE) $\wedge$ (DER Availability $=$ TRUE) \\
\hline (Interruption $=$ TRUE) $\wedge($ Alert = NULL) \\
\hline$($ New Pitch $<$ Old Pitch $) \wedge\left(\right.$ Microgrid Demand $\left.>\mu_{d}+\epsilon_{d}\right)$ \\
\hline$($ New Pitch $>$ Old Pitch $) \wedge$ (Microgrid Demand $\left.<\mu_{d}-\epsilon_{d}\right)$ \\
\hline
\end{tabular}

sensors. Construct the DNF predicate for each device type by joining the corresponding Table IV, V or VI expressions with a disjunction. For clarity, the DNF predicate was left unreduced; clauses in the DNF predicate are traced to behavior rules easily. This makes it evident that attacks interact through common state variables with the same logical clause. While it will yield a more elegant expression and maybe a more efficient implementation, reducing the DNF predicate would obscure the traceability of the logical clauses and interdependence of the behavior rules.

d) Identify State Components and Component Ranges: Continuous components are quantized at integer scale in permissible ranges. For example, system demand is in the range of $[0,1000 \mathrm{GW}]$ and duty cycle is in the range of $[0$, $100 \%$ ]. Table VII shows a complete list of the permissible ranges of state components. The resulting $\mathrm{HE}$ automaton has $2 \times 1001 \times 101 \times 100 \times 2 \times 2 \times 2 \times 2 \times 2 \times 2=1.294 \times 10^{9}$ states. The resulting DAP automaton has $1001 \times 1001 \times 2 \times$ $2 \times 11 \times 11 \times 2 \times 2 \times 2 \times 2=7.759 \times 10^{9}$ states. The resulting SEM automaton has $2^{32} \times 2 \times 100 \times 2 \times 1001 \times 2 \times 2 \times 2 \times$ $2 \times 91 \times 1001=2.506 \times 10^{21}$ states. All of these automata are too large; this state explosion is dealt with in the next step.

TABLE VI

SEM BAD BEHAVIOR INDICATORS IN CONJUNCTIVE NORMAL FORM

\begin{tabular}{|l|}
\hline Time $>$ Usage.Timestamp $+\epsilon$ \\
\hline$($ Appliance $=$ ACTIVE $) \wedge\left(\right.$ Billing Rate $\left.>\mu_{r}+\epsilon_{r}\right)$ \\
\hline$\left(\right.$ Energy Storage $=$ FALSE $\wedge\left(\right.$ Billing Rate $\left.<\mu_{r}-\epsilon_{r}\right)$ \\
\hline$($ Appliance $=$ ACTIVE $) \wedge\left(\right.$ Microgrid Demand $\left.>\mu_{d}+\epsilon_{d}\right)$ \\
\hline$($ Energy Storage $=$ FALSE $) \wedge\left(\right.$ Microgrid Demand $\left.<\mu_{d}-\epsilon_{d}\right)$ \\
\hline$($ DER Connection $=$ FALSE $) \wedge($ DER Availability $=$ TRUE $)$ \\
\hline$($ Interruption $=$ TRUE $) \wedge($ Alert $=$ NULL $)$ \\
\hline$($ New Pitch $<$ Old Pitch $) \wedge\left(\right.$ Subscriber Demand $\left.>\mu_{d}+\epsilon_{d}\right)$ \\
\hline$($ New Pitch $>$ Old Pitch $) \wedge\left(\right.$ Subscriber Demand $\left.<\mu_{d}-\epsilon_{d}\right)$ \\
\hline
\end{tabular}

TABLE VII

MODERN ELECTRICITY INFRASTRUCTURE STATE COMPONENTS

\begin{tabular}{|lll|}
\hline Name & $\begin{array}{l}\text { Control or } \\
\text { Reading }\end{array}$ & Range \\
\hline \hline Appliance Block Activation & Control & true, false \\
System Demand & Reading & {$[0,1000 \mathrm{GW}]$} \\
Appliance Duty Cycle & Control & {$[0,100 \%]$} \\
Billing Rate & Control & $(0,1 \mathrm{USD} / \mathrm{kWh}]$ \\
Switch Position & Control & open, closed \\
Fault & Reading & false, true \\
Maintenance & Control & false, true \\
DER Connection & Control & false, true \\
Interruption & Reading & false, true \\
Alert & Control & false, true \\
Load Request & Control & {$[0,1000 \mathrm{~kW}]$} \\
Microgrid Demand & Reading & {$[0,1000 \mathrm{MW}]$} \\
Island & Control & false, true \\
Forwarded Packets & Reading & {$[0,10 / \mathrm{s}]$} \\
Received Packets & Reading & {$[0,10 / \mathrm{s}]$} \\
DER Availability & Reading & false, true \\
Wind DER Generator Pitch & Control & {$\left[0,90^{\circ}\right]$} \\
Usage Age & Reading & {$\left[0,2^{32}\right]$} \\
Appliance Activation & Control & false, true \\
Energy Storage & Control & false, true \\
Subscriber Demand & Reading & {$[0,1000 \mathrm{~kW}]$} \\
\hline
\end{tabular}

e) Manage State Space: To manage the number of states, the size of the state machine is reduced by abbreviating the values for some components. Only three values are relevant for system, microgrid and subscriber demand: below threshold, normal and above threshold. Therefore, the domains for these components are collapsed to three values for the HE, DAP and SEM, respectively. This treatment yields a modest HE state machine with $2 \times 3 \times 3 \times 3 \times 2 \times 2 \times 2 \times 2 \times 2 \times 2=3456$ states, out of which 1008 are identified as safe states and 2448 are unsafe states. Only two values are relevant for networking: whether or not packets forwarded and packets received differ by more than some threshold. Therefore, the domain for this component is collapsed to two values. This treatment yields a modest DAP state machine with $3 \times 3 \times 2 \times 2 \times 2 \times 2 \times 2 \times 2 \times 3=1728$ states, out of which 255 are identified as safe states and 1473 are unsafe states. Only three values are relevant for rate: below threshold, normal and above threshold. Also, only two values are relevant for usage reporting: current or missing. Therefore, the domains for these components are collapsed to three and two values, respectively. This treatment yields a modest SEM state machine with $2 \times 2 \times 3 \times 2 \times 3 \times 2 \times 2 \times 2 \times 2 \times 3=3456$ states, out of which 396 are identified as safe states and 3060 are unsafe states.

\section{E. Collect Compliance Degree Data}

BRIDS relies on the use of monitor nodes, e.g., an SEM or a DAP is a monitor node of another SEM. The monitor node knows the state machine of the trustee node assigned to it. The monitor node periodically measures the amount of time the trustee node stays in safe and unsafe states as the trustee node migrates from one state to another triggered by events causing state transitions. A binary grading policy, i.e., assigning a compliance degree of 1 to a safe state and 0 to an unsafe state, is considered. Let $c$ be the compliance degree of a device. The compliance degree $c$ of a device essentially is equal to the proportion of the time the device is in safe states. 
Let $\mathcal{S}$ be the set of safe states the trustee node traverses over a period of time $T$. Let $t_{i}$ be the sojourn time that the trustee node stays in a safe state $i$, as measured by the monitor node. Then the monitor node collects an instance of $c$ by:

$$
c=\frac{\sum_{i \in \mathcal{S}} t_{i}}{T}
$$

If a node stays only in safe states during $T$, then by Equation 1 , its compliance degree $c$ is one. On the other hand, if a node stays only in unsafe states only during $T$, then its compliance degree $c$ is zero. The monitor node monitors and collects the trustee node's compliance degree history $c_{1}, c_{2}, \ldots, c_{n}$ for $n$ monitoring periods, where $n$ is sufficiently large, based on which it concludes whether or not the trustee node is compromised.

The state machines generated are leveraged to collect compliance degree data of a good and a bad node. With Equation 1 , the compliance degree $c$ is essentially equal to the sum of the probabilities of safe states i.e., $c=\sum_{j \in \mathcal{S}} \pi_{j}$, where $\pi_{j}$ is the limiting probability that the node is in state $j$ of the state machine and $\mathcal{S}$ is the set of safe states in the state machine. Compliance degree history $c_{1}, c_{2}, \ldots, c_{n}$ of a node is then collected by means of Monte Carlo simulation. That is, given a good (or a bad) node's state machine, start from state 0 and then follow the stochastic process of this node as it goes from one state to another. This is continued until at least one state is reentered sufficiently often (say 100 times). Then $\pi_{j}$ is calculated using the ratio of the number of transitions leading to state $j$ to the total number of state transitions. Then one instance of compliance degree is collected. A sufficiently large $n$ test runs was repeated to collect $c_{1}, c_{2}, \ldots, c_{n}$ needed for computing the distribution of the compliance degree of a good or a bad node performing reckless or random attacks.

\section{F. Compliance Degree Distribution}

The measurement of compliance degree of a device frequently is not perfect and can be affected by noise and unreliable wireless communication in the WAN, NAN and HAN segments. The compliance degree is modeled by a random variable $X$ with $G(\cdot)=\operatorname{Beta}(\alpha, \beta)$ distribution [18], with the value 0 indicating that the output is totally unacceptable (zero compliance) and 1 indicating the output is totally acceptable (perfect compliance), such that $G(a), 0 \leq a \leq 1$, is given by

$$
G(a)=\int_{0}^{a} \frac{\Gamma(\alpha+\beta)}{\Gamma(\alpha) \Gamma(\beta)} x^{\alpha-1}(1-x)^{\beta-1} d x
$$

and the expected value of $X$ is given by

$$
E_{B}[X]=\int_{0}^{1} x \frac{\Gamma(\alpha+\beta)}{\Gamma(\alpha) \Gamma(\beta)} x^{\alpha-1}(1-x)^{\beta-1} d x=\frac{\alpha}{\alpha+\beta}
$$

The $\alpha$ and $\beta$ parameters are to be estimated based on the method of maximum likelihood by using the compliance degree history collected $\left(c_{1}, c_{2}, \ldots, c_{n}\right)$ during the system's testing phase. The maximum likelihood estimates of $\alpha$ and $\beta$ are obtained by numerically solving the following equations:

$$
\frac{n \frac{\partial \Gamma(\hat{\alpha}+\hat{\beta})}{\partial \hat{\alpha}}}{\Gamma(\hat{\alpha}+\hat{\beta})}-\frac{n \frac{\partial \Gamma(\hat{\alpha})}{\partial \hat{\alpha}}}{\Gamma(\hat{\alpha})}+\sum_{i=1}^{n} \log c_{i}=0
$$

$$
\frac{n \frac{\partial \Gamma(\hat{\alpha}+\hat{\beta})}{\partial \hat{\beta}}}{\Gamma(\hat{\alpha}+\hat{\beta})}-\frac{n \frac{\partial \Gamma(\hat{\beta})}{\partial \hat{\beta}}}{\Gamma(\hat{\alpha})}+\sum_{i=1}^{n} \log \left(1-c_{i}\right)=0
$$

where

$$
\frac{\partial \Gamma(\hat{\alpha}+\hat{\beta})}{\partial \hat{\alpha}}=\int_{0}^{\infty}(\log x) x^{\hat{\alpha}+\hat{\beta}-1} e^{-x} d x .
$$

A less general, though simpler model, is to consider a single parameter $\operatorname{Beta}(\beta)$ distribution with $\alpha$ equal to 1 . In this case, the density is $\beta(1-x)^{\beta-1}$ for $0 \leq x \leq 1$ and 0 otherwise. The maximum likelihood estimate of $\beta$ is

$$
\hat{\beta}=\frac{n}{\sum_{i=1}^{n} \log \left(\frac{1}{1-c_{i}}\right)}
$$

The reason the Beta distribution is chosen is that the domain of the Beta distribution can be viewed as a probability, so it can be used to describe the prior distribution over the probability (of a distribution) which models the node compliance degree. By applying Bayesian inference, the Beta distribution then can be used as the posterior distribution of the probability after observing sufficient instances.

\section{G. False Negative and Positive Probabilities}

Our intrusion detection technique is characterized by false negative and false positive probabilities, denoted by $p_{\mathrm{fn}}$ and $p_{\mathrm{fp}}$, respectively. A false negative occurs when a bad node is missed as a good device, while a false positive occurs when a good node is misdiagnosed as a bad device. While neither is desirable, a false negative is especially impactful to the system's continuity of operation. In this paper, a threshold criterion is considered. That is, if a bad node's compliance degree denoted by $X_{b}$ with a probability distribution obtained by Equation 2 is higher than a system minimum compliance threshold $C_{T}$, then there is a false negative. Suppose that the compliance degree $X_{b}$ of a bad node is modeled by a $G(\cdot)=\operatorname{Beta}(\alpha, \beta)$ distribution. Then the host IDS false negative probability $p_{\mathrm{fn}}$ is given by:

$$
p_{\text {fn }}=\operatorname{Pr}\left\{X_{b}>C_{T}\right\}=1-G\left(C_{T}\right) .
$$

On the other hand, if a good node's compliance degree denoted by $X_{g}$ is less than $C_{T}$, then there is a false positive. Again suppose that the compliance degree $X_{g}$ of a good node is modeled by a $G(\cdot)=\operatorname{Beta}(\alpha, \beta)$ distribution. Then the host false positive probability $p_{\mathrm{fp}}$ is given by:

$$
p_{\text {fp }}=\operatorname{Pr}\left\{X_{g} \leq C_{T}\right\}=G\left(C_{T}\right) .
$$

\section{NUMERICAL DATA}

Numerical data is reported in this section. A sequence of compliance degree values $\left(c_{1}, c_{2}, \ldots, c_{n}\right)$ is first collected for a good or bad device based on Monte Carlo simulation. Equation 5 is then applied to compute the $\beta$ parameter value of $G(\cdot)=\operatorname{Beta}(\alpha, \beta)$ for the probability distribution of the compliance degree for a good device or a bad device performing random attacks. $p_{\mathrm{fn}}$ and $p_{\mathrm{fp}}$ are then calculated by Equations 6 and 7, respectively. The minimum compliance 
TABLE VIII

$\beta$ IN Beta $(1, \beta)$ AND RESUlting $p_{\mathrm{fn}}$ AND $p_{\mathrm{fp}}$ VAlues Under VARIOUS ATTACK MODELS FOR HE $\left(C_{T}=0.90\right)$

\begin{tabular}{|l|rrr|}
\hline Attack Type & $\beta$ & $p_{\text {fn }}$ & $p_{\text {fp }}$ \\
\hline Random $\left(p_{a}=1.00\right)$ & 99.5 & $<0.001 \%$ & $2.30 \%$ \\
Random $\left(p_{a}=0.80\right)$ & 4.33 & $0.0047 \%$ & $2.30 \%$ \\
Random $\left(p_{a}=0.40\right)$ & 1.10 & $7.99 \%$ & $2.30 \%$ \\
Random $\left(p_{a}=0.20\right)$ & 0.633 & $23.3 \%$ & $2.30 \%$ \\
Random $\left(p_{a}=0.10\right)$ & 0.449 & $35.5 \%$ & $2.30 \%$ \\
Random $\left(p_{a}=0.05\right)$ & 0.353 & $44.3 \%$ & $2.30 \%$ \\
\hline
\end{tabular}

TABLE IX

$\beta$ IN BetA $(1, \beta)$ AND RESUlting $p_{\text {fn }}$ AND $p_{\text {fp }}$ VAlues Under VARIOUS ATTACK MODELS FOR DAP $\left(C_{T}=0.90\right)$

\begin{tabular}{|l|rrr|}
\hline Attack Type & $\beta$ & $p_{\mathrm{fn}}$ & $p_{\mathrm{fp}}$ \\
\hline Random $\left(p_{a}=1.00\right)$ & 49.6 & $<0.001 \%$ & $4.59 \%$ \\
Random $\left(p_{a}=0.80\right)$ & 4.19 & $0.0064 \%$ & $4.59 \%$ \\
Random $\left(p_{a}=0.40\right)$ & 1.10 & $7.89 \%$ & $4.59 \%$ \\
Random $\left(p_{a}=0.20\right)$ & 0.644 & $22.7 \%$ & $4.59 \%$ \\
Random $\left(p_{a}=0.10\right)$ & 0.464 & $34.3 \%$ & $4.59 \%$ \\
Random $\left(p_{a}=0.05\right)$ & 0.372 & $42.5 \%$ & $4.59 \%$ \\
\hline
\end{tabular}

threshold $C_{T}$ is then adjusted to control $p_{\mathrm{fn}}$ and $p_{\mathrm{fp}}$ obtainable. With $p_{\text {err }}$ a monitor node can misidentify the status the trustee node is in. $p_{\text {err }}$ is set to $0.010,0.015$ and 0.020 for HE, DAP and SEM nodes, respectively. This is because 1 - $2 \%$ of mis-monitoring due to ambient noise and wireless communication faults in these environments is reasonable. This is based on Lin and Latchman reporting a $0.11-2.04 \%$ Power Line Communication packet error rate [15] and Hong et al. reporting a 0.02 - $4 \%$ failure rate [11]. The mis-monitoring error probability of an SEM toward another SEM is higher than that of a DAP toward another DAP, or an HE toward another HE because of limited range and capability of an SEM device.

Tables VIII, IX and $\mathrm{X}$ show the $\beta$ values and the resulting $p_{\text {fn }}$ and $p_{\mathrm{fp}}$ values when $C_{T}$ is $0.9\left(C_{T}\right.$ is a design parameter to be fine-tuned to trade high false positives for low false negatives). Because the expected compliance degree following a $\operatorname{Beta}(\alpha, \beta)$ distribution is $\alpha /(\alpha+\beta)$ as given by Equation 3 , it is seen that $\beta$ is close to 0 for a good node or a hidden bad node with a low attack probability (e.g., $p_{a}=0.05$ ) since such a node will have the average compliance degree close to 1. On the other hand, $\beta$ is much larger than 0 for a bad node with a high attack probability (e.g., $p_{a}=1$ ) since such a node will have the average compliance degree much lower than 1 .

It is observed that when the random attack probability $p_{a}$ is high, the attacker can be easily detected as evidenced by a low false negative probability. Especially when $p_{a}=1$, a reckless attacker can hardly be missed. On the other hand, as

TABLE X

$\beta$ IN Beta $(1, \beta)$ AND Resulting $p_{\text {fn }}$ AND $p_{\text {fp }}$ Values Under Various ATTACK MODELS FOR SEM $\left(C_{T}=0.90\right)$.

\begin{tabular}{|l|rrr|}
\hline Attack Type & $\beta$ & $p_{\mathrm{fn}}$ & $p_{\mathrm{fp}}$ \\
\hline Random $\left(p_{a}=1.00\right)$ & 32.8 & $<0.001 \%$ & $6.87 \%$ \\
Random $\left(p_{a}=0.80\right)$ & 4.06 & $0.0086 \%$ & $6.87 \%$ \\
Random $\left(p_{a}=0.40\right)$ & 1.11 & $7.78 \%$ & $6.87 \%$ \\
Random $\left(p_{a}=0.20\right)$ & 0.656 & $22.1 \%$ & $6.87 \%$ \\
Random $\left(p_{a}=0.10\right)$ & 0.479 & $33.2 \%$ & $6.87 \%$ \\
Random $\left(p_{a}=0.05\right)$ & 0.390 & $40.7 \%$ & $6.87 \%$ \\
\hline
\end{tabular}

$p_{a}$ decreases, the attacker becomes more hidden and insidious, and the false negative probability increases. The false positive probability remains the same regardless of the random attack probability because it is not related to the attacker behavior.

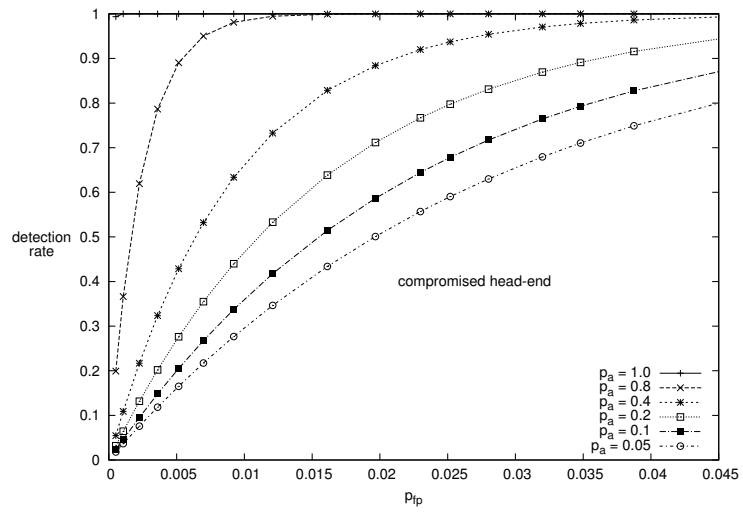

Fig. 2. HE Receiver Operating Characteristic Graph.

By adjusting $C_{T}$, the specification-based IDS technique can effectively trade higher false positives for lower false negatives to cope with more sophisticated and hidden random attackers. This is especially desirable for ultra safe and secure applications for which a false negative may have a dire consequence. Figure 2 shows a Receiver Operating Characteristic (ROC) graph of intrusion detection rate (i.e., $1-p_{\mathrm{fn}}$ ) versus false positive probability $\left(p_{\mathrm{fp}}\right)$ obtained as a result of adjusting $C_{T}$. In Figure 2 there are several curves for each node type, one for each random attacker case with a different attack probability $p_{a}$. As $C_{T}$ is increased, the detection rate increases (vertically up on a ROC graph) while the false probability increases (toward the right of a ROC graph). It is seen that with the specification-based IDS technique, the detection rate of the node can approach $100 \%$ for detecting attackers, that is, an attacker is always detected with probability 1 without false negatives, while bounding the false positive probability to below $0.2 \%$ for reckless attackers and below $6 \%$ for random attackers.

\section{Comparative Analysis}

The performance of BRIDS is compared with contemporary anomaly-based IDSs for HEs, DAPs and SEMs, including CLONALG and AIRS2Parallel [22], [23], LOUD, LOED, LOUD-GLR and LOED-GLR [10].

Zhang et al. [23] reported that CLONALG had a false positive rate of $0.7 \%$ and a false negative rate of $21.02 \%$ and AIRS2Parallel had a false positive rate of $1.3 \%$ and a false negative rate of $26.32 \%$. Zhang et al. [22] further compared the effectiveness of audit data from three sources: home IDS (HIDS), neighborhood IDS (NIDS) and wide-area IDS (WIDS). These three approaches correspond with the SEM, DAP and HE nodes identified in Figure 1. Here the authors reported that CLONALG had an accuracy of $99.70 \%$ for HEs, $80.10-97.00 \%$ for DAPs and $93.90-99.30 \%$ for SEMs. They reported that AIRS2Parallel had an accuracy of $91.50 \%$ for HEs, $82.10-96.10 \%$ for DAPs and $95.10-98.70 \%$ 
TABLE XI

COMPARISON RESUlTS FOR HE.

\begin{tabular}{|l|l|l|}
\hline Approach & Detection Accuracy & Device \\
\hline \hline CLONALG [23] & $100-0.7-21.02=78.28 \%$ & $\mathrm{HE}$ \\
\hline AIRS2Parallel [23] & $100-1.3-26.32=78.38 \%$ & $\mathrm{HE}$ \\
\hline LOUD [10] & $100-0.1-9=90.90 \%$ & $\mathrm{HE}$ \\
\hline LOED [10] & $100-0.1-16=83.90 \%$ & $\mathrm{HE}$ \\
\hline LOUD-GLR [10] & $100-0.1-5=94.90 \%$ & $\mathrm{HE}$ \\
\hline LOED-GLR [10] & $100-0.1-9=90.90 \%$ & $\mathrm{HE}$ \\
\hline CLONALG WIDS [22] & $99.70 \%$ & $\mathrm{HE}$ \\
\hline AIRS2Parallel WIDS [22] & $91.50 \%$ & $\mathrm{HE}$ \\
\hline BRIDS $\left(C_{T}=0.50\right)$ & $100-0.70-0.00=99.30 \%$ & $\mathrm{HE}$ \\
\hline BRIDS $\left(C_{T}=0.73\right)$ & $100-1.30-0.00=98.70 \%$ & $\mathrm{HE}$ \\
\hline BRIDS $\left(C_{T}=0.09\right)$ & $100-0.10-0.01=99.89 \%$ & $\mathrm{HE}$ \\
\hline
\end{tabular}

TABLE XII

COMPARISON RESULTS FOR DAP

\begin{tabular}{|l|l|l|}
\hline Approach & Detection Accuracy & Device \\
\hline \hline CLONALG NIDS [22] & {$[80.10,97.00 \%]$} & DAP \\
\hline AIRS2Parallel NIDS [22] & {$[82.10,96.10 \%]$} & DAP \\
\hline BRIDS $\left(C_{T}=0.37\right)$ & $100-0.70-0.00=99.30 \%$ & DAP \\
\hline BRIDS $\left(C_{T}=0.58\right)$ & $100-1.30-0.00=98.70 \%$ & DAP \\
\hline BRIDS $\left(C_{T}=0.06\right)$ & $100-0.10-1.68=98.22 \%$ & DAP \\
\hline
\end{tabular}

for SEMs. The authors provided no $p_{\mathrm{fn}}$ or $p_{\mathrm{fp}}$ information, but presumably the worst detection accuracy is obtained when $p_{\mathrm{fp}}$ is very low. He and Blum [10] investigated LOUD, LOED, LOUD-GLR and LOED-GLR approaches to anomaly-based IDS. They fixed the false positive probability (i.e., $p_{\mathrm{fp}}$ ) at $0.1 \%$ and showed that the detection rate (i.e., $1-p_{\mathrm{fn}}$ ) for each approach varies over a wide range based on the parameterization. The LOUD-GLR approach reportedly performs the best with the detection accuracy of $100-0.1-5=94.9 \%$.

Tables XI, XII and XIII summarize the comparative performances among contemporary IDSs for HE, DAP and SEM devices, respectively. The performance metric is detection accuracy defined as $1-p_{\mathrm{fp}}-p_{\mathrm{fn}}$. For cases where $p_{\mathrm{fn}}$ and $p_{\mathrm{fp}}$ are reported [10], [23], the detection accuracy value is shown following the $1-p_{\mathrm{fp}}-p_{\mathrm{fn}}$ format. For cases where $p_{\mathrm{fn}}$ and $p_{\mathrm{fp}}$ are not reported [22], the detection accuracy value or a range of detection accuracy values is shown only. For comparison, the adversary is configured with $p_{a}=1$ (reckless attacks). BRIDS performance is shown for $C_{T}=0.50$ for $\mathrm{HE}, C_{T}=0.37$ for DAP and $C_{T}=0.29$ for SEM to approximate the CLONALG $p_{\text {fp }}$ of $0.7 \%$ [23]. BRIDS performance is shown for $C_{T}=0.73$ for HE, $C_{T}=0.58$ for DAP and $C_{T}=0.47$ for SEM to approximate the AIRS2Parallel $p_{\mathrm{fp}}$ of $1.3 \%$ [23]. BRIDS performance is shown for $C_{T}=0.09$ for $\mathrm{HE}, C_{T}=0.06$ for DAP and $C_{T}=0.05$ for SEM to approximate the LOUD, LOED, LOUD-GLR and LOED-GLR $p_{\mathrm{fp}}$ of $0.1 \%$ [10].

Tables XI, XII and XIII support the claim that by effectively

TABLE XIII

COMPARISON RESULTS FOR SEM.

\begin{tabular}{|l|l|l|}
\hline Approach & Detection Accuracy & Device \\
\hline \hline CLONALG HIDS [22] & {$[93.90,99.30 \%]$} & SEM \\
\hline AIRS2Parallel HIDS [22] & {$[95.10,98.70 \%]$} & SEM \\
\hline BRIDS $\left(C_{T}=0.29\right)$ & $100-0.70-0.00=99.30 \%$ & SEM \\
\hline BRIDS $\left(C_{T}=0.47\right)$ & $100-1.30-0.00=98.70 \%$ & SEM \\
\hline BRIDS $\left(C_{T}=0.05\right)$ & $100-0.10-7.82=92.08 \%$ & SEM \\
\hline
\end{tabular}

adjusting $C_{T}$ to trade false positives for low false negatives, BRIDS outperforms existing anomaly-based IDS approaches, especially for HE and DAP devices.

\section{CONCLUSIONS}

For a modern electrical grid, being able to detect attackers while limiting the false positive probability to protect the continuity of operation is of utmost importance. In this paper, a behavior-rule specification-based IDS technique for intrusion detection of physical devices was proposed. The utility by head-ends, distribution access points/data aggregation points and subscriber energy meters was exemplified. This study also demonstrated that the detection probability approaches one (that is, the attacker can always be caught without false negatives) while bounding the false positive probability to below $0.2 \%$ for reckless attackers and below $6 \%$ for random attackers (that is, the probability of misidentifying a good node as a bad node can always be bounded to a very low level). Through a comparative analysis, it was demonstrated a behavior-rule specification-based IDS technique outperforms existing anomaly-based IDS approaches for detecting intruders.

Two future research directions extending from this study are (a) investigating and analyzing intrusion response and repair strategies [17]; and (b) implementing behavior rules on applications. Possible intrusion responses include evicting individual compromised nodes, isolating compromised segments (microgrid or larger scope) and adjusting IDS parameters (e.g., $T_{\mathrm{IDS}}, m$ and $C_{T}$ ) to increase detection strength. Possible repair strategies are to identify compromised segments and for each one: stop operating, revert all nodes to certified software loads and configurations, rekey/reset passwords and progressively resume operation from the production side of the network towards the subscribers. Possible implementation strategies are to encode the state machine, host IDS software and system IDS software in a high-level language, cross-compile for the targets of interest, deploy and tune the parameterization (e.g., $T_{\mathrm{IDS}}, m$ and $C_{T}$ ) based on desired versus actual false negative and positive rates. Another future research direction is to investigate other intrusion detection criteria [1], [4], [5] based on accumulation of deviation from good states in addition to the current binary criterion used in the paper based on a minimum compliance threshold to further improve the detection rate without compromising the false positive probability.

\section{REFERENCES}

[1] F. B. Bastani, I. R. Chen, and T. W. Tsao. Reliability of Systems with Fuzzy-Failure Criterion. In Annual Reliability and Maintainability Symposium, pages 442-448, Anaheim, CA, USA, January 1994.

[2] R. Berthier and W. Sanders. Specification-based intrusion detection for advanced metering infrastructures. In 2011 IEEE 17th Pacific Rim International Symposium on Dependable Computing, pages 184-193, December 2011.

[3] A. A. Cárdenas, S. Amin, Z.-S. Lin, Y.-L. Huang, C.-Y. Huang, and S. Sastry. Attacks against process control systems: risk assessment, detection, and response. In The 6th ACM Symposium on Information, Computer and Communications Security, pages 355-366, Hong Kong, China, March 2011. 
[4] I. R. Chen and F. B. Bastani. Effect of artificial-intelligence planningprocedures on system reliability. IEEE Transactions on Reliability, 40(3):364-369, 1991.

[5] I. R. Chen, F. B. Bastani, and T. W. Tsao. On the reliability of AI planning software in real-time applications. IEEE Transactions on Knowledge and Data Engineering, 7(1):4-13, 1995.

[6] Y. Chen and B. Luo. S2a: secure smart household appliances. In The second ACM conference on Data and Application Security and Privacy, pages 217-228, San Antonio, TX, USA, February 2012.

[7] S. Cheung, B. Dutertre, M. Fong, U. Lindqvist, K. Skinner, and A. Valdes. Using model-based intrusion detection for SCADA networks. In SCADA Security Scientific Symposium, pages 127-134, Miami, FL, USA, January 2007.

[8] A. da Silva. Decentralized intrusion detection in wireless sensor networks. In 1st ACM inter. workshop on quality of service \& security in wireless and mobile networks, pages 16-23, 2005.

[9] B. Dutertre. Formal modeling and analysis of the modbus protocol. Critical Infrastructure Protection, pages 189-204, 2007.

[10] Q. He and R. S. Blum. Smart grid monitoring for intrusion and fault detection with new locally optimum testing procedures. In 2011 IEEE International Conference on Acoustics, Speech and Signal Processing, pages 3852-3855, May 2011.

[11] Y.-F. Hong, Z.-Q. Liu, H.-X. Yin, and J.-H. Zhang. A new method for smart grid reliability. In 2011 Asia-Pacific Power and Energy Engineering Conference, pages 1-4, March 2011.

[12] K. Ioannis, T. Dimitriou, and F. Freiling. Towards intrusion detection in wireless sensor networks. In 13th European Wireless Conference, 2007.

[13] P. Jokar, H. Nicanfar, and V. Leung. Specification-based intrusion detection for home area networks in smart grids. In 2011 IEEE International Conference on Smart Grid Communications, pages 208213, October 2011.

[14] R. Klump and M. Kwiatkowski. Distributed ip watchlist generation for intrusion detection in the electrical smart grid. Critical Infrastructure Protection IV, 342:113-126, 2010.

[15] Y.-J. Lin and H. Latchman. On the effects of maximum transmission unit in power line communication networks. In IEEE International Symposium on Power Line Communications and Its Applications, pages 511-516, March 2007.

[16] B. Luitel, G. Venayagamoorthy, and C. Johnson. Enhanced wide area monitoring system. In Innovative Smart Grid Technologies, 2010, pages $1-7$, January 2010.

[17] R. Mitchell and I. R. Chen. Effect of Intrusion Detection and Response on Reliability of Cyber Physical Systems. IEEE Transactions on Reliability, 62(1):199-210, March 2013.

[18] S. M. Ross. Introduction to Probability Models, 10th Edition. Academic Press, 2009.

[19] C.-W. Ten, J. Hong, and C.-C. Liu. Anomaly detection for cybersecurity of the substations. IEEE Transactions on Smart Grid, 2(4):865-873, December 2011.

[20] X. Wang and P. Yi. Security framework for wireless communications in smart distribution grid. IEEE Transactions on Smart Grid, 2(4):809-818, December 2011

[21] Y. Wang, D. Ruan, J. Xu, M. Wen, and L. Deng. Computational intelligence algorithms analysis for smart grid cyber security. In Y. Tan, Y. Shi, and K. Tan, editors, Advances in Swarm Intelligence, volume 6146 of Lecture Notes in Computer Science, pages 77-84. 2010.

[22] Y. Zhang, L. Wang, W. Sun, R. Green, and M. Alam. Artificial immune system based intrusion detection in a distributed hierarchical network architecture of smart grid. In 2011 IEEE Power and Energy Society General Meeting, pages 1-8, Detroit, MI, USA, July 2011.

[23] Y. Zhang, L. Wang, W. Sun, R. Green, and M. Alam. Distributed intrusion detection system in a multi-layer network architecture of smart grids. IEEE Transactions on Smart Grid, 2(4):796-808, December 2011. 